\title{
Effort thrombosis: A case study and discussion
}

\author{
Robert Matthew Collins (MB BCh, MSc Sports Med)) ${ }^{1}$ \\ Dina Christina Janse van Rensburg (MMed (PhysMed) (Rheumatology), MSc (Sports Med), MB ChB) ${ }^{1}$ \\ Catherina Cornelia Grant (BSc, BSc Hon (Physiology), MSc (Physiology)) ${ }^{1}$ \\ Jonathan Speridon Patricios (MB BCh, MMed (Sci), FACSM, FFSEM (UK)) ${ }^{1}$ \\ Martin Veller (MMed (Surg), FCS(SA)) ${ }^{2}$ \\ Dimakatso Althea Ramagole (MB ChB, MSc Sports Med)) ${ }^{1}$ \\ ${ }^{1}$ Section Sports Medicine, University of Pretoria \\ 2 University of the Witwatersrand, Johannesburg
}

\section{Summary}

Paget-Schroetter syndrome or effort thrombosis is a rare condition with potentially severe consequences, affecting young and otherwise healthy athletes. Its classic clinical presentation should be rapidly identified to promote early diagnosis and treatment. This prevents recurrences and long-term complications such as persistent swelling of the affected arm. In a 1-year period we saw two young male athletes with this condition. One was treated conservatively and the other surgically, with vastly different outcomes. We therefore present their cases as well as a review of relevant literature to emphasise treatment of this rare condition.

\section{Case 1}

A 23-year-old male professional road cyclist presented 2 days after an acute onset of swelling of the right arm. He had been given a new bicycle by his sponsor, and had gone for a 4-hour cycle. His position on the new bike was slightly different to his previous cycling position, with the handlebars being slightly closer to his body, and he noticed swelling and heaviness of his right arm the following day. On examination, his right arm was markedly swollen from the axilla to the fingers, with the biceps diameter being $30 \mathrm{~cm}$ on the right compared with $27 \mathrm{~cm}$ on the left. He had normal pulses and no adverse neurological findings. A duplex Doppler of the arm revealed no thrombus formation, and the veins were reported as having normal compressibility. A venogram was then conducted, which showed a deep vein thrombosis (DVT) of the right subclavian vein, related to the medial third of the clavicle and first rib with early collateral formation. Risk factors for thrombus formation include a malignant melanoma which had been excised 13 years previously, a high haematocrit $(49.4 \%)$, the cyclist's posture, and the new cycling

\section{CORRESPONDENCE:}

Dr Robert Matthew Collins

University of Pretoria, Section Sports Medicine

PO Box 11

Mondeor

2110 Johannesburg

Tel: +27 116805684

Fax: +27 865167975

E-mail: robcollins@wol.co.za position adopted on the new bicycle. Blood investigations included a thrombotic work-up, which was completely normal. His X-rays of chest and cervical spine were both normal. There was no evidence of recurrence of the melanoma.

Treatment with anticoagulation was initiated within 2 days of his initial presentation. He was offered thrombolysis of the clot, but refused this treatment. Thrombolysis is usually performed by insertion of a percutaneous catheter, and a haemolytic agent is infiltrated directly into the site of the clot. The procedure is usually performed at the same time as the venogram, as the same catheter may be used for both procedures.

When his swelling persisted beyond 7 days he requested further intervention, and was offered surgical excision of the affected vein with first rib resection to decompress the thoracic outlet. His surgery was performed 2 weeks after the initial presentation, and included the above-mentioned treatment and a saphenous vein graft to anastamose the subclavian vein. Unfortunately, several intraoperative complications were encountered including a laceration of the pleura, haemothorax, on-table severe bradycardia presumed to be caused by a pulmonary embolus and requiring resuscitation, and severing of the phrenic nerve.

The patient managed to overcome these complications, continued anticoagulant therapy for 6 months, and then attempted unsuccessfully to return to professional cycling. He was followed up a year later, and found to have no residual swelling of the arm.

\section{Case 2}

Case 2 was a 25 -year-old amateur rugby prop forward. He described a tearing sound in his left shoulder, which occurred when he performed a spear tackle (an action that he described as being similar to a dead-lift in weight lifting) on another player. He presented 2 weeks later in the consulting rooms with marked swelling and heaviness of his right arm. The diameters of his arms at biceps level were 47 $\mathrm{cm}$ and $42 \mathrm{~cm}$ respectively. The pulses of his right arm felt weak due to the swelling of the arm, and he had no neurological deficit in the arm. He was markedly tender to palpation and swollen over the right coracoid process. The initial Doppler of his subclavian and axillary vein showed no abnormality, but when it was repeated by an experienced vascular surgeon, it showed a DVT at the junction of the subclavian and jugular veins on the right side. Anticoagulant therapy and a pressure stocking of the arm were initiated at his first visit. He was offered thrombolysis, but he too refused the treatment. He was treated with physical therapy for a torn pectoralis minor muscle that 
was thought to have caused the thrombosis, and postural correction. At 3-month follow-up he had no residual swelling of his arm, and was scheduled to undergo positional venography to assess the patency of the subclavian vein and exclude thoracic outlet compression of the vein when he was lost to follow-up.

He has since been contacted, is completely symptom free, and has no recurrent swelling of his arm at all.

\section{Discussion}

DVT is a common condition, but only 2 - $4 \%$ of all DVTs involve the upper limb. ${ }^{1-6}$ Primary subclavian vein thrombosis is a relatively uncommon condition, affecting 2/100 000 people per year, which represents $15-24 \%$ of all upper limb DVTs, ${ }^{7,8}$ but it is the most common vascular disorder affecting professional, collegiate or highschool athletes. $5,7,9$

It was first described by Sir James Paget in 1858, and later by Leold van Schroetter in 1884. The English surgeon, Hughes, gave it the eponym Paget-Schroetter syndrome (PSS) when he reviewed 320 cases from literature in 1949. ${ }^{2,3,5-8,10,11}$ Another synonym for the condition in literature is 'effort thrombosis', which emphasises the causal relationship between certain forms of exercise and thrombosis of the subclavian vein. ${ }^{4,6}$ This relationship was first identified by Von Schroetter in 1899 . $^{2,8}$

PSS occurs primarily in young, otherwise healthy subjects (mean age 15 - 30 years) who participate in repetitive upper limb activities. ${ }^{3,4,7-10}$ It occurs twice as frequently in men as in women, affecting the right or dominant arm in $80 \%$ of cases. ${ }^{1,2,5,6,8}$ It has been reported in many sports, including golf, American football, weight lifting, baseball, wrestling, tennis, martial arts, backpacking, billiards, swimming, rowing and cheerleading. ${ }^{1-3,10}$ Because of the youth and otherwise healthy state of those presenting with this condition, it is often ignored or misdiagnosed. ${ }^{8}$ In a series of eight patients with the condition, Zell et al. found that five of the eight patients were untrained athletes for whom exercise was not the norm. ${ }^{2}$

The mechanism of thrombosis is through a thoracic outlet compression and/or repetitive intimal strain of the axillary or subclavian vein by retroversion and hyperabduction of the arm. ${ }^{7,9}$ Repetitive intimal strain can lead to inflammation and traumatic fissures in the intima of the vein, resulting ultimately in thrombus formation. $2,4,6,10,11$ Compression of the vein in the thoracic outlet through lateral abduction of the arm or hypertrophy of subclavian or anterior scalene muscles can cause turbulence in the vein, which is exacerbated by increased venous flow experienced during exercise. ${ }^{2,10}$ Thrombosis occurs most commonly at the level of the subclavian vein where it crosses the thoracic outlet and may be compressed. ${ }^{4}$ Lifting of heavy weights can also cause compression of the vein in the thoracic outlet by depression of the shoulder, causing compression of the vein between the clavicle and first rib. 8,10

The occurrence of PSS can also be contributed to by a hypercoagulable state ${ }^{10}$ and upper limb thromboses are more frequently associated with underlying malignant disorders than those in the lower limb. ${ }^{11}$ Melby et al. stated that the chronic repetitive compression and intimal micro-trauma that result in PSS make it an acute manifestation of a chronic condition rather than a single traumatic event. ${ }^{9}$ This observation is important in the management of the condition, as it is essential to treat the causative pathology to prevent recurrence. ${ }^{9}$

PSS should be distinguished aetiologically from other subclavian DVTs, which are caused by primary diseases and other known risk factors such as malignant neoplasms, treatment with central venous catheters or pacemakers, or cervical ribs. ${ }^{2}$ These patients develop secondary thrombosis, and are older, and have concurrent systemic illness. $^{3}$

The anatomical relations of the subclavian vein in the thoracic outlet are:

- superior and anterior: the clavicle, subclavius muscle and costoclavicular ligament

- inferior: first rib

- posterior: anterior scalene muscle. ${ }^{1,3,8}$

Other structures which are in close relation to the subclavian vein in and around the thoracic outlet include: pectoralis minor and supraspinatus muscles, coracocostal ligament, osseous exostoses, cervical ribs, fibrous cords. ${ }^{3,8}$ Cervical ribs are present in between $0.5 \%$ and $1 \%$ of the general population, but cause symptoms in less than $10 \%$ of these people, most of whom have arterial or neurological thoracic outlet syndromes.

Patients present with symptoms of venous obstruction of the arm including a dull aching pain, non-oedematous swelling, a feeling of heaviness in the arm, and bluish discolouration of the arm. $^{6-8,10}$ Symptoms range in severity, may be position-dependent, and occasionally the patient may be asymptomatic. ${ }^{7,8}$ Differential diagnosis includes:

- fracture of the mid-shaft of the humerus

- soft-tissue injury or infection with compartment syndrome

- muscular strain or sprain resulting in local tenderness and swelling. 6

Pulmonary thrombo-embolism is a rare complication of PSS, and occurs in between $7 \%$ and $20 \%$ of cases. ${ }^{3-5,7}$

Early and accurate diagnosis is of paramount importance to prevent long-term complications and recurrence. ${ }^{2,3}$ It has been stated that with PSS the earlier the diagnosis and treatment, the better the outcome. ${ }^{11}$ Duplex Doppler is the first diagnostic test of choice, as it is non-invasive, and is reported as having a high sensitivity and specificity for peripheral DVT. ${ }^{7}$ Melby et al., however, reported a relatively low diagnostic sensitivity of $71 \%$ of duplex Doppler to upper extremity thrombosis, ${ }^{9}$ and Shebel states that it is only reliable for thrombus extending into the axillary vein. ${ }^{8}$ They stated that this is as a result of technical difficulties in visualising the subclavian vein below the clavicle, especially in the presence of extensive collateral veins, and the inability to compress the vein within the thoracic outlet. $^{8,9}$ They therefore suggested that a negative duplex Doppler should not exclude thrombosis, and that contrast venography should be the diagnostic test of choice. ${ }^{1,6,8,9}$ Positional venography with the arm in neutral, abducted and externally rotated positions, is also of value in not only assessing costoclavicular compression of the subclavian vein, but also compression of collateral veins with positional changes of the arm. ${ }^{8}$

There is no consensus on the optimal treatment of PSS, as most published data consist of individual case reports and small series. ${ }^{3,5,11}$ Hiken and Ameli conducted a literature review to determine the most appropriate treatment for PSS, and concluded that since treatments, follow-up and conclusions reported were so varied, optimal treatment remains controversial. ${ }^{12}$ Traditional treatment consists of anticoagulation and elevation of the limb to facilitate thrombus resolution, and prevent further thrombus formation. ${ }^{3,7}$ There are varying reports of the incidence of post-thrombotic syndrome (PTS), which is characterised by chronic swelling and heaviness of the arm and hand. Incidences of PTS of between $41 \%$ and $73 \%{ }^{3-6,11}$ with conservative treatment have been reported, with severity of 
symptoms ranging vastly. Historically most authors have concluded, in the light of these varying data, that non-operative treatment of axillary thrombosis provided a predictably good outcome, and that surgical intervention was not warranted. ${ }^{3}$

Despite these conclusions, there was persistent concern over residual symptoms following PSS treated with anticoagulation therapy alone, which 'rarely produces symptom-free use of the arm, and imposes limitations that appear unacceptable to most patients'. 9 This led to the advent of catheter-directed thrombolysis, which is currently regarded as the initial treatment of choice, ${ }^{5}$ as a treatment. ${ }^{3,10}$ As mentioned previously, this involves injection of a thrombolytic agent into the site of the thrombus via a percutaneous catheter. The procedure is often performed at the time of venography, as the same catheter can be used for both procedures. The advantage of early thrombolysis is that it restores venous patency, thus minimising the damage to the endothelium, and reducing the risk of post-thrombotic syndrome. $^{3,7}$

Venous patency is accepted as the main factor in preventing residual symptoms. ${ }^{3}$ This has been shown to be achieved through thrombolysis alone if the treatment is initiated within 1 week of the onset of symptoms. 5 F, 13 Fibrinolysis alone is unsuccessful in nearly $20 \%$ of patients even when undertaken within a week of onset of symptoms. ${ }^{5}$ There is a greater incidence of fibrous stricture formations at the site of the thrombus if thrombolysis occurs beyond 1 week. In these cases, further surgical intervention such as vein patch angioplasty and decompression of the thoracic outlet is indicated. ${ }^{13}$

Other methods of overcoming strictures have been tried, including balloon angioplasty and the placement of stents. Balloon angioplasty is usually unsuccessful due to extrinsic compression of the subclavian vein and dense perivenous scar tissue, which results in immediate recoil or re-stenosis. ${ }^{5,9}$ Endoluminal stents are similarly an ineffective treatment, as subclavian vein compression frequently results in bending or fracture of the stent with subsequent re-thrombosis.

Defining whether or not the patient has a pre-existing anatomical anomaly is essential in determining which patients will benefit from surgical decompression of the thoracic outlet, as PSS cannot be effectively treated without addressing compressive mechanisms causing thrombogenesis. ${ }^{5,7}$ If extrinsic venous compression is identified, many surgeons advocate early decompression of the thoracic outlet via resection of the first rib or clavicle. Others, however, recommend a trial of conservative therapy after thrombolysis. ${ }^{7}$ Hicken et al. stated that surgical intervention should be reserved for patients in whom there is a specific indication. ${ }^{12}$

In a series of 32 cases of PSS treated between January 1997 and January 2007, Melby et al. showed excellent results to surgical intervention. ${ }^{9}$ They offered surgical treatment to all patients. Their treatment included thoracic outlet decompression via complete anterior and middle scalenectomy, brachial plexus neurolysis, excision of subclavius muscle tendon, and resection of the first rib. They also performed external venolysis of the subclavian vein on all patients, excising all scar tissue from the axillary vein to the subclavianjugular-innominate vein junction. They were able to demonstrate normal compressibility of the vein through palpation or intraoperative venography in $56 \%$ of the patients. These patients had no further venous reconstruction. The remaining $44 \%$ had residual narrowing of the subclavian vein in at least one portion. The affected section of the vein was resected, and reconstructed using an autologous saphenous vein patch. In $59 \%$ of the patients they also created a radiocephalic arteriovenous fistula, which assisted in maintaining flow through the grafted area. ${ }^{9}$ The median time to full return to competitive sport in their series was 3.5 months from the time of surgery (range $2-10$ months), with $50 \%$ of the patients resuming sports participation by 3 months. ${ }^{9}$ They found that there was no difference in time to return to sporting activities after operative intervention in patients with delayed diagnosis or recurrent symptoms. ${ }^{9}$

\section{Conclusion}

PSS is a rare condition, but the most common vascular condition affecting athletes. It may present with subtle symptoms, but early diagnosis is imperative in being able to initiate early treatment. Duplex Doppler, although the first diagnostic test of choice, is unable to always definitively exclude the condition, and venography is recommended if there is clinical suspicion and a negative Doppler test. Anticoagulation is important to prevent thrombus proliferation, but is often unsuccessful as a sole treatment for PSS. Thrombolysis is often successful in restoring venous patency and preventing longterm sequelae of post-thrombotic syndrome if performed within 7 days of the onset of the condition. Surgical intervention may be required to restore venous patency and compressibility and to relieve external compression of the vein in the thoracic outlet. These can be assessed through positional venography in the patient who has completely symptomatically recovered with conservative treatment. Failure to achieve complete recovery with conservative treatment alone is an indication for further intervention. Surgical options include external venolysis, resection of affected segments of vein with saphenous vein patching, and thoracic outlet decompression via first rib resection or scalene or subclavian muscle resection.

\section{REFERENCES}

1. Toya N, Fujita T, Ohki T. Push-up exercise induced thrombosis of the subclavian vein in a young woman: report of a case. Surg Today 2007;37(12):1093-1095.

2. Zell L, Kindermann W, Marschall F, Scheffler P, Gross J, Buchter A. PagetSchroetter syndrome in sports activities--case study and literature review. Angiology 2001:52(5):337-342.

3. DiFelice GS, Paletta GA,Jr, Phillips BB, Wright RW. Effort thrombosis in the elite throwing athlete. Am J Sports Med 2002;30(5):708-712.

4. Meier MA, Rubenfire M. Life-threatening acute and chronic thromboembolic pulmonary hypertension and subclavian vein thrombosis. Clin Cardiol 2006;29(3):103-106.

5. Feugier P, Aleksic I, Salari R, Durand X, Chevalier JM. Long-term results of venous revascularization for Paget-Schroetter syndrome in athletes. Ann Vasc Surg 2001;15(2):212-218.

6. Chaudhry MA, Hajarnavis J. Paget-von Schrotter syndrome: primary subclavian-axillary vein thrombosis in sport activities. Clin J Sport Med 2003;13(4):269-271.

7. Roche-Nagle G, Ryan R, Barry M, Brophy D. Effort thrombosis of the upper extremity in a young sportsman: Paget-Schroetter syndrome. $\mathrm{Br} \mathrm{J}$ Sports Med 2007;41(8):540-541.

8. Shebel ND, Marin A. Effort thrombosis (Paget-Schroetter syndrome) in active young adults: current concepts in diagnosis and treatment. J Vascular Nursing 2006;24(4):116-126.

9. Melby SJ, Vedantham S, Narra VR, et al. Comprehensive surgical management of the competitive athlete with effort thrombosis of the subclavian vein (Paget-Schroetter syndrome). J Vasc Surg 2008;47(4):809-820.

10. McGlinchey PG, Shamsuddin SA, Kidney JC. Effort-induced thrombosis of the subclavian vein--a case of Paget-Schroetter syndrome. Ulster Med J 2004;73(1):45-46.

11. Oktar GL, Ergul EG. Paget-Schroetter syndrome. Hong Kong Med J 2007;13(3):243-245.

12. Hicken GJ, Ameli FM. Management of subclavian-axillary vein thrombosis: a review. Canad J Surg 1998;41(1):13-25.

13. Molina JE. Need for emergency treatment in subclavian vein effort thrombosis. J Am Coll Surg 1995;181(5):414-420. 